\title{
THE AXIAL VECTOR CURRENT ANOMALY AND THE MESON DECAY CONSTANTS FOR THE IMPROVED LATTICE FERMION ACTION
}

\author{
Herbert W. HAMBER and Chi Min WU \\ The Institute for Advanced Study, Princeton, NJ 08540, USA
}

Received 15 November 1983

We discuss the anomaly in the axial vector current for the improved fermion action on the lattice. A detailed discussion of the fermion regularization dependence of the anomaly is given and it is shown that for finite lattice spacing the improved fermion action gives a more reasonable value for the anomaly contribution at finite quark mass than the Wilson action. We also present some results on the perturbative corrections to the decay constants of mesons for the improved fermion action.

Introduction. In a previous paper [1] we proposed an improved fermion action on the lattice by adding a next nearest neighbor interaction term to the generalized Wilson fermion action [2]. We gave arguments there which suggested that the proposed action should approach the continuum limit more rapidly, and suggested to investigate whether theoretically motivated improvements would also lead to improvements in the quality of the numerical results.

In this paper we address the question of the behavior of the axial vector current anomaly for the improved fermion action for small quark masses, and elucidate the role of the species doubling fermions in reproducing the correct anomaly contribution in the continuum. This problem is of importance because of the well known connection between the anomaly and the $\eta^{\prime}$ mass. We show that for finite and not too small quark mass (in lattice units) the coefficient of the anomaly has a strong dependence on the fermion regularization. The suppression of the anomaly contribution for the Wilson fermion action at finite mass will be shown to be significantly reduced when the improved fermion action is used. Further on we compute the perturbative correction factors for the meson decay constants to lowest order. These factors are useful in comparing the decay constants on the lattice with those in the continuum.

1. The axial anomaly for the improved action. It has been recognized since a long time that the spontaneous breaking of chiral symmetry is a comerstone in the understanding of low-lying hadron spectroscopy [3]. It is also of course, an important topic in the context of the lattice gauge theory.

It was pointed out by several authors [4-6] that the Wilson fermion action reproduces the correct anomaly in the continuum limit.

In our previous paper [1] we proposed an improvement action of the following form

$S=S_{\text {gauge }}+S_{\text {fermion }}$

with

$S_{\text {gauge }}=\frac{1}{g^{2}} \sum_{n, \mu<\nu} \operatorname{tr}\left[U_{n, \mu} U_{n+\mu, \nu} U_{n+\nu, \mu}^{+} U_{n, \nu}^{+}+\right.$h.c. -2$]$, 


$$
\begin{aligned}
& S_{\text {fermion }}=k \sum_{n, \mu}\left[\bar{\psi}_{n}\left(r-\gamma_{\mu}\right) U_{n, \mu} \psi_{n+\mu}+\bar{\psi}_{n+\mu}\left(r+\gamma_{\mu}\right) U_{n, \mu}^{+} \psi_{n}\right]+\sum_{n, \mu}\left[\bar{\psi}_{n}\left(C-D \gamma_{\mu}\right) U_{n, \mu} U_{n+\mu, \mu} \psi_{n+2 \mu}\right. \\
& \left.\quad+\bar{\psi}_{n+2 \mu}\left(C+D \gamma_{\mu}\right) U_{n+\mu, \mu}^{+} U_{n, \mu}^{+} \psi_{n}\right]-\sum_{n} \bar{\psi}_{n} \psi_{n} .
\end{aligned}
$$

This action gave the Feynman rules for the fermion propagator and the three-point fermion-gluon vertices in the form

$$
\begin{aligned}
& S_{\mathrm{F}}^{-1}(p)=1-2 k \sum_{\mu}\left(r \cos p_{\mu}-\mathrm{i} \gamma_{\mu} \sin p_{\mu}\right)-2 \sum_{\mu}\left(C \cos 2 p_{\mu}-\mathrm{i} D \gamma_{\mu} \sin 2 p_{\mu}\right), \\
& V_{\mu}^{(3)}(p, q)=2 g\left[k r \sin \frac{1}{2}(p+q)_{\mu}+\mathrm{i} k \gamma_{\mu} \cos \frac{1}{2}(p+q)_{\mu}\right. \\
& \left.\quad+2 C \sin (p+q)_{\mu} \cos \frac{1}{2}(p-q)_{\mu}+2 \mathrm{i} D \gamma_{\mu} \cos (p+q)_{\mu} \cos \frac{1}{2}(p-q)_{\mu}\right] T^{a} .
\end{aligned}
$$

Here the $T^{a}$ 's are the generators of $\mathrm{SU}(N)$ and the hopping parameter $k$ is related to the fermion mass. We then require that the fermion propagator and the vertices approach the continuum limit rapidly, which is achieved by adjusting the coefficients of the $\mathrm{O}\left(p^{2}\right)$ and of the $\mathrm{O}\left(p^{3}\right)$ terms in the fermion propagator to be zero. In this way we get the constraint

$C=-\frac{1}{4} k r, \quad D=-\frac{1}{8} k$.

Notice that what we have written down is basically a truncated SLAC derivative fermion action, in the sense that an improvement to all orders in $p$ would correspond just to using the (nonlocal) SLAC derivative formulation [7].

We will now discuss the axial anomaly for the improved fermion action. By performing the chiral transformation on the fermion fields

$\psi_{n} \rightarrow \exp \left(\mathrm{i} \gamma_{5} \theta\right) \psi_{n}, \quad \bar{\psi}_{n} \rightarrow \bar{\psi}_{n} \exp \left(\mathrm{i} \gamma_{5} \theta\right)$,

we get the lattice axial Ward identity

$\sum_{\mu}\left[J_{\mu, n}^{5(1)}-J_{\mu, n-\mu}^{5(1)}+\frac{1}{2}\left(J_{\mu, n}^{5(2)}-J_{\mu, n-2 \mu}^{5(2)}\right)\right]-2 m J_{n}^{5}-X_{n}=0$,

where we have defined

$$
\begin{aligned}
& J_{\mu, n}^{5(1)}=k\left[\bar{\psi}_{n} \gamma_{\mu} \gamma_{5} U_{n, \mu} \psi_{n+\mu}+\bar{\psi}_{n+\mu} \gamma_{\mu} \gamma_{5} U_{n, \mu}^{+} \psi_{n}\right] \\
& J_{\mu, n}^{5(2)}=-\frac{1}{4} k\left[\bar{\psi}_{n} \gamma_{\mu} \gamma_{5} U_{n, \mu} U_{n+\mu, \mu} \psi_{n+2 \mu}+\bar{\psi}_{n+2 \mu} \gamma_{\mu} \gamma_{5} U_{n+\mu, \mu}^{+} U_{n, \mu}^{+} \psi_{n}\right] \\
& m=(2 / 3 k)(1-6 k r), \quad J_{n}^{5}=(3 k / 2) \bar{\psi}_{n} \gamma_{5} \psi_{n}, \\
& X_{n}=12 k r \bar{\psi}_{n} \gamma_{5} \psi_{n}-k r \sum_{\mu}\left[\bar{\psi}_{n} \gamma_{5} U_{n, \mu} \psi_{n+\mu}+\bar{\psi}_{n-\mu} \gamma_{5} U_{n-\mu, \mu} \psi_{n}+\bar{\psi}_{n} \gamma_{5} U_{n-\mu, \mu}^{+} \psi_{n-\mu}+\bar{\psi}_{n+\mu} \gamma_{5} U_{n, \mu}^{+} \psi_{n}\right] \\
& \quad+\frac{1}{4} k r \sum_{\mu}\left[\bar{\psi}_{n} \gamma_{5} U_{n, \mu} U_{n+\mu, \mu} \psi_{n+2 \mu}+\bar{\psi}_{n-2 \mu} \gamma_{5} U_{n-2 \mu, \mu} U_{n-\mu, \mu} \psi_{n}\right. \\
& \left.\quad+\bar{\psi}_{n} \gamma_{5} U_{n-\mu, \mu}^{+} U_{n-2 \mu, \mu}^{+} \psi_{n-2 \mu}+\bar{\psi}_{n+2 \mu} \gamma_{5} U_{n+\mu, \mu}^{+} U_{n, \mu}^{+} \psi_{n}\right]
\end{aligned}
$$

The V-V-A three-current matrix element is defined as

$$
\sum_{n, m} \exp [-\mathrm{i}(p n+q m)]\left\langle 0\left|\mathrm{~T}\left(J_{\mu, n}^{a} J_{\nu, m}^{b} J_{\alpha, 0}^{5(i)}\right)\right| 0\right\rangle=\Gamma_{\alpha \mu \nu}^{a b(i)}(p, q)+\Gamma_{\alpha \nu \mu}^{b a(i)}(q, p),
$$




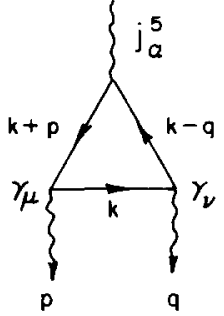

a

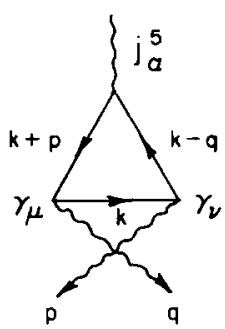

b
Fig. 1. (a), (b) The lowest order contribution to the $\mathrm{V}-\mathrm{V}-\mathrm{A}$ three-curren matrix element.

and

$\Gamma_{\alpha \mu \nu}^{a b}(p, q)+\Gamma_{\alpha \nu \mu}^{b a}(q, p)=\sum_{i=1}^{2} \Gamma_{\alpha \mu \nu}^{a b(i)}(p, q)+\sum_{i=1}^{2} \Gamma_{\alpha \nu \mu}^{b a(i)}(q, p)$.

The quantity $J_{\mu, n}^{a}$ is the vector current

$J_{\mu, n}^{a}=g k\left[\bar{\psi}_{n}\left(r-\gamma_{\mu}\right) T^{a} \psi_{n+\mu}-\bar{\psi}_{n+\mu}\left(r+\gamma_{\mu}\right) T^{a} \psi_{n}\right]$

$-g k\left[\bar{\psi}_{n}\left(\frac{1}{4} r-\frac{1}{8} \gamma_{\mu}\right) T^{a} \psi_{n+2 \mu}+\bar{\psi}_{n-\mu}\left(\frac{1}{4} r-\frac{1}{8} \gamma_{\mu}\right) T^{a} \psi_{n+\mu}-\bar{\psi}_{n+\mu}\left(\frac{1}{4} r+\frac{1}{8} \gamma_{\mu}\right) T^{a} \psi_{n-\mu}-\bar{\psi}_{n+2 \mu}\left(\frac{1}{4} r+\frac{1}{8} \gamma_{\mu}\right) T^{a} \psi_{n}\right]$.

The lowest order contribution to this matrix element on the lattice is given by (see fig. $1 \mathrm{a}$ and $1 \mathrm{~b}$ )

$$
\begin{aligned}
& \Gamma_{\alpha \mu \nu}^{a b}(p, q)+\Gamma_{\alpha \nu \mu}^{b a}(q, p)=-2 k \operatorname{tr}\left(T^{a} T^{b}\right) \int_{-\pi}^{\pi} \frac{\mathrm{d}^{4} k}{(2 \pi)^{4}} \operatorname{tr}\left\{\gamma_{\alpha} \gamma_{5}\left[\cos \frac{1}{2}(2 k+2 l+p-q)_{\alpha}-\frac{1}{4} \cos (2 k+2 l+p-q)_{\alpha}\right]\right. \\
& \left.\quad \times S_{\mathrm{F}}(k+p+l) V_{\mu}^{(3)}(k+p+l, k+l) S_{\mathrm{F}}(k+l) V_{\nu}^{(3)}(k+l, k-q+l) S_{\mathrm{F}}(k-q+l)\right\}+(p \leftrightarrow q, \mu \leftrightarrow \nu),
\end{aligned}
$$

where we have shifted the internal momentum $k_{\mu} \rightarrow k_{\mu}+l_{\mu}$, and $l_{\mu}$ can be taken to be a linear combination of the external momenta

$l_{\mu}=c p_{\mu}+d q_{\mu}$.

The coefficients $c$ and $d$ will be determined later in order to satisfy the vector Ward identity. We also wish to define the following quantities

$\sum_{n, m} \exp [-\mathrm{i}(p n+q m)]\left\langle 0\left|\mathrm{~T}\left(J_{\mu, n}^{a} J_{\nu, m}^{b} 2 m J_{0}^{5}\right)\right| 0\right\rangle=2 m \Gamma_{\mu \nu}^{a b}(p, q)+2 m \Gamma_{\nu \mu}^{b a}(q, p)$,

and

$\sum_{n, m} \exp [-\mathrm{i}(p n+q m)]\left\langle 0\left|\mathrm{~T}\left(J_{\mu, n}^{a} J_{\nu, m}^{b} X_{0}\right)\right| 0\right\rangle=X_{\mu \nu}^{a b}(p, q)+X_{\nu \mu}^{b a}(q, p)$.

The lowest order contributions to $2 m \Gamma_{\mu \nu}^{a b}(p, q)$ and $X_{\mu \nu}^{a b}(p, q)$ are given by

$$
\begin{aligned}
& 2 m \Gamma_{\mu \nu}^{a b}(p, q)=-\frac{3}{2} k \operatorname{tr}\left(T^{a} T^{b}\right) \int_{-\pi}^{\pi} \frac{\mathrm{d}^{4} k}{(2 \pi)^{4}} \operatorname{tr}\left[2 m \gamma_{5} S_{\mathrm{F}}(k+p+l)\right. \\
& \left.\quad \times V_{\mu}^{(3)}(k+p+l, k+l) S_{\mathrm{F}}(k+l) V_{\nu}^{(3)}(k+l, k-q+l) S_{\mathrm{F}}(k-q+l)\right],
\end{aligned}
$$

and by 


$$
\begin{aligned}
& X_{\mu \nu}^{a b}(p, q)=-k \operatorname{tr}\left(T^{a} T^{b}\right) \int_{-\pi}^{\pi} \frac{\mathrm{d}^{4} k}{(2 \pi)^{4}} \operatorname{tr}\left\{\gamma_{5}[\bar{M}(k+p+l)+\bar{M}(k-q+l)] S_{\mathrm{F}}(k+p+l)\right. \\
& \left.\times V_{\mu}^{(3)}(k+p+l, k+l) S_{\mathrm{F}}(k+l) V_{\nu}^{(3)}(k+l, k-q+l) S_{\mathrm{F}}(k-q+l)\right\},
\end{aligned}
$$

where we have set for brevity

$\bar{M}(k)=6 r-2 r \sum_{\rho}\left(\cos k-\frac{1}{4} \cos 2 k\right)_{\rho}$.

In this way we can give the deviation in one loop level from the tree axial Ward identity of eq. (1.6). In momentum space, it can be written as

$T_{\mu \nu}^{a b}(p, q)=$

$$
\begin{aligned}
& \sum_{\alpha}\left\{1-\exp \left[-\mathrm{i}(p+q)_{\alpha}\right]\right\} \Gamma_{\alpha \mu \nu}^{a b(1)}(p, q)+\frac{1}{2} \sum_{\alpha}\left\{1-\exp \left[-2 \mathrm{i}(p+q)_{\alpha}\right]\right\} \Gamma_{\alpha \mu \nu}^{a b(2)}(p q)-2 m \Gamma_{\mu \nu}^{a b}(p, q)-X_{\mu \nu}^{a b}(p, q) \\
& =k \operatorname{tr}\left(T^{a} T^{b}\right) \cdot \int_{-\pi}^{\pi} \frac{\mathrm{d}^{4} k}{(2 \pi)^{4}} \operatorname{tr}\left\{\gamma_{5}[M(k+l+p)+\mathrm{i} \not \delta(k+l+p)]+\left[M(k-q+l)+\mathrm{i} S(k-q+l) \gamma_{5}\right]\right. \\
& \left.\times S_{\mathrm{F}}(k+p+l) V_{\mu}^{(3)}(k+p+l, k+l) S_{\mathrm{F}}(k+l) V_{\nu}^{(3)}(k+l, k-q+l) S_{\mathrm{F}}(k-q+l)\right\}
\end{aligned}
$$

where

$M(k)=\frac{3}{2} m+6 r-2 r \sum_{\rho}\left(\cos k-\frac{1}{4} \cos 2 k\right)_{\rho}=\bar{M}(k)+\frac{3}{2} m$,

and

$\not(k)=2 \sum_{\mu} \gamma_{\mu}\left(\sin k-\frac{1}{8} \sin 2 k\right)_{\mu}$.

After taking the trace, we obtain

$$
\begin{aligned}
& T_{\mu \nu}^{a b}(p, q)+T_{\nu \mu}^{b a}(q, p)=-16 g^{2} \operatorname{tr}\left(T^{a} T^{b}\right) \epsilon_{\alpha \mu \beta \nu} \int_{-\pi}^{\pi} \frac{\mathrm{d}^{4} k}{(2 \pi)^{4}}\left[\cos \frac{1}{2}(2 k+p+2 l)_{\mu}-\frac{1}{4} \cos (2 k+p+2 l)_{\mu} \cos \frac{1}{2} p_{\mu}\right] \\
& \quad \times\left[\cos \frac{1}{2}(2 k+2 l-q)_{\nu}-\frac{1}{4} \cos (2 k+2 l-q)_{\nu} \cos \frac{1}{2} q_{\nu}\right] \frac{S_{\beta}(k+l)}{M^{2}(k+l)+S^{2}(k+l)} \\
& \quad \times\left(\frac{S_{\alpha}(k+l+p)}{M^{2}(k+l+p)+S^{2}(k+l+p)}-\frac{S_{\alpha}(k+l-q)}{M^{2}(k+l-q)+S^{2}(k+l-q)}\right)+(p \leftrightarrow q, \mu \leftrightarrow \nu),
\end{aligned}
$$

where we have set

$S_{\mu}(k)=2\left(\sin k-\frac{1}{8} \sin 2 k\right)_{\mu}, \quad S^{2}(k)=4 \sum_{\mu}\left(\sin k-\frac{1}{8} \sin 2 k\right)_{\mu}^{2}$.

We now expand (1.21) in powers of the external momenta and keep terms up to second order $T_{\mu \nu}^{a b}(p, q)+T_{\nu \mu}^{b a}(q, p)=\left(g^{2} / 4 \pi^{2}\right) \operatorname{tr}\left(T^{a} T^{b}\right) \epsilon_{\alpha \mu \beta \nu} p_{\alpha} q_{\beta}(c-d) I(r)$,

where $l(r)$ is the integral 
$I(r)=\lim _{m \rightarrow 0} \frac{32}{\pi^{2}} \int_{-\pi}^{\pi} \mathrm{d}^{4} k \frac{M(k) \Pi_{\lambda=1}^{4}\left(\cos k-\frac{1}{4} \cos 2 k\right)_{\lambda} \tilde{M}(k)}{\left[M^{2}(k)+S^{2}(k)\right]^{3}}$,

$\tilde{M}(k)=\frac{3}{2} m+6 r-2 r \sum_{\rho}^{4} \frac{\left(\cos k-\frac{1}{4} \cos 2 k\right)_{\rho}^{2}+\left(\sin k-\frac{1}{8} \sin 2 k\right)_{\rho}\left(\sin k-\frac{1}{2} \sin 2 k\right)_{\rho}}{\left(\cos k-\frac{1}{4} \cos 2 k\right)_{\rho}}$.

In a similar way we get the vector Ward identity

$\sum_{\mu} \mathrm{i} p_{\mu}\left[\Gamma_{\alpha \mu \nu}^{a b}(p, q)+\Gamma_{\alpha \nu \mu}^{b a}(q, p)\right]=\left(g^{2} / 8 \pi^{2}\right) \operatorname{tr}\left(T^{a} T^{b}\right)(c-d+2) \epsilon_{\alpha \rho \nu \sigma} p_{\rho} q_{\sigma} I(r)$.

The total axial anomaly contribution is then

$X_{\mu \nu}^{a b}(p, q)+X_{\nu \mu}^{b a}(q, p)=\left(g^{2} / 2 \pi^{2}\right) \operatorname{tr}\left(T^{a} T^{b}\right) \epsilon_{\mu \alpha \nu \beta} p_{\alpha} q_{\beta} \tilde{I}(r)$,

where we have defined

$\widetilde{I}(r)=\lim _{m \rightarrow 0} \frac{32}{\pi^{2}} \int_{-\pi}^{\pi} \mathrm{d}^{4} k \frac{\bar{M}(k) \Pi_{\rho}\left(\cos k-\frac{1}{4} \cos 2 k\right)_{\rho} \widetilde{M}(k)}{\left[M^{2}(k)+S^{2}(k)\right]^{3}}$.

The coefficients $c$ and $d$ are determined by requiring that the vector Ward identity be satisfied

$d-c=2$.

Now a very careful treatment for the anomaly coefficients $I(r)$ and $\widetilde{I}(r)$ is needed. Generally speaking, the regularization parameter $\alpha$ can be sent to zero only after the momentum integration has been performed. This means that in the integrand of the expressions in eqs. (1.24) and (1.28), the parameter $m$ goes to zero but cannot be set to zero.

To proceed further we divide momentum space into 16 regions and calculate each fermion species contribution to the anomaly separately. It is known that in the ordinary (nearest neighbor) generalized Wilson fermion action (the action of eq. (1.2) with the next nearest neighbor terms) one has on the lattice 16 fermion species instead of the one expected in the continuum. All of them except one have a mass of the order of the cutoff. In Wilson case, at $k=k_{\mathrm{c}}$ the particles at $p a=[\pi, 0,0,0]$ have a mass $2 r / a$ (and there are 4 of them), at $[\pi, \pi, 0,0]$ a mass $4 r / a(6)$, at $[\pi, \pi, \pi, 0]$ a mass $6 r / a(4)$, and the one at $[\pi, \pi, \mathrm{k}, \pi]$ a mass $8 r / a(1)$.

Here, as in the ordinary Wilson case, the different species are classified in 5 categories with multiplicities $C_{0}=1, C_{1}=4, C_{2}=6, C_{3}=4, C_{4}=1$. The total anomaly can be decomposed into its 5 species contributions and written as

$I(r)=\sum_{j=0}^{4} C_{j} B_{j}(r)$

and analogously

$\tilde{I}(r)=\sum_{j=0}^{4} C_{j} \widetilde{B}_{j}(r)$

with

$B_{j}(r)=\lim _{m \rightarrow 0} A_{j}(r, m)$,

$A_{j}(r, m)=\frac{32}{\pi^{2}} \int_{-\pi / 2}^{\pi / 2} \mathrm{~d}^{4} k \widetilde{T}_{j} \frac{M(k) \Pi_{\lambda=1}^{4}\left(\cos k-\frac{1}{4} \cos 2 k\right)_{\lambda} \tilde{M}(k)}{\left[M^{2}(k)+S^{2}(k)\right]^{2}}$, 
$\widetilde{B}_{j}(r)=\lim _{m \rightarrow 0} \widetilde{A}_{j}(r, m)$,

$\widetilde{A}_{j}(r, m)=\frac{32}{\pi^{2}} \int_{-\pi / 2}^{\pi / 2} \mathrm{~d}^{4} k \widetilde{T}_{j} \frac{\bar{M}(k) \Pi_{\lambda=1}^{4}\left(\cos k-\frac{1}{4} \cos 2 k\right)_{\lambda} \widetilde{M}(k)}{\left[M^{2}(k)+S^{2}(k)\right]^{2}}$.

The momentum shift operator $\widetilde{T}_{j}$ is defined as

$\widetilde{T}_{j} f\left(k_{1}, k_{2}, \ldots k_{j}, k_{j+1}, \ldots\right)=f\left(k_{1}+\pi, k_{2}+\pi, \ldots k_{j}+\pi, k_{j+1}, \ldots\right)$,

with $\widetilde{T}_{0}=1$ and $j=0,1,2,3,4 . \widetilde{A}_{j}(r, m)$ is a continuous function of $m$ and we have $\widetilde{B}_{j}(r)=\widetilde{A}_{j}(r, m=0)$. By using the following identity (valid for any value of $m$ )

$\left(1+\frac{1}{4} \sum_{\rho=1}^{4} \frac{\left(\sin k-\frac{1}{8} \sin 2 k\right)_{\rho}}{\left(\cos k-\frac{1}{4} \cos 2 k\right)_{\rho}} \frac{\mathrm{d}}{\mathrm{d} k_{\rho}}\right) \frac{1}{\left[M^{2}(k)+S^{2}(k)\right]^{2}}=\frac{M(k) \tilde{M}(k)}{\left[M^{2}(k)+S^{2}(k)\right]^{3}}$,

one can easily show that the integrand of $A_{j}(r, m)$ and $\widetilde{A}_{j}(r, m=0)$ can be expressed as a total derivative

$B_{j}(r)=\lim _{m \rightarrow 0} \frac{8}{\pi^{2}} \int_{-\pi / 2}^{\pi / 2} \mathrm{~d}^{4} k \widetilde{T}_{j} \sum_{\lambda=1}^{4} \frac{\mathrm{d}}{\mathrm{d} k_{\lambda}}\left(\frac{\left(\sin k-\frac{1}{8} \sin 2 k\right)_{\lambda} \Pi_{\sigma \neq \lambda}\left(\cos k-\frac{1}{4} \cos 2 k\right)_{\sigma}}{\left[M^{2}(k)+S^{2}(k)\right]^{2}}\right)$,

$\widetilde{B}_{j}(r)=\frac{8}{\pi^{2}} \int_{-\pi / 2}^{\pi / 2} \mathrm{~d}^{4} k \widetilde{T}_{j} \sum_{\lambda=1}^{4} \frac{\mathrm{d}}{\mathrm{d} k_{\lambda}}\left(\left.\frac{\left(\sin k-\frac{1}{8} \sin 2 k\right)_{\lambda} \Pi_{\sigma \neq \lambda}\left(\cos k-\frac{1}{4} \cos 2 k\right)_{\sigma}}{\left[M^{2}(k)+S^{2}(k)\right]^{2}}\right|_{m=0}\right)$.

After integrating over one momentum in (1.38) and in (1.39) we get

$\widetilde{B}_{0}=B_{0}-1=\pi^{-2} 4 J_{0}-1, \quad \widetilde{B}_{1}=B_{1}=-\pi^{-2}\left(J_{0}-3 J_{1}\right), \quad \widetilde{B}_{2}=B_{2}=-\pi^{-2}\left(2 J_{1}-2 J_{2}\right)$,

$\widetilde{B}_{3}=B_{3}=-\pi^{-2}\left(3 J_{2}-J_{3}\right), \quad \widetilde{B}_{4}=B_{4}=-\pi^{-2} 4 J_{3}$,

where we have set

$J_{i}=16 \int_{-\pi / 2}^{\pi / 2} \mathrm{~d}^{3} k \widetilde{T}_{i} \frac{\Pi_{\lambda=1}^{3}\left(\cos k-\frac{1}{4} \cos 2 k\right)_{\lambda}}{\left\{\left[\frac{3}{2} m+\frac{11}{2} r-2 r \Sigma_{\rho}^{3}\left(\cos k-\frac{1}{4} \cos 2 k\right)_{\rho}^{2}\right]^{2}+4+4 \Sigma_{\rho}^{3}\left(\sin k-\frac{1}{8} \sin 2 k\right)_{\rho}^{2}\right\}^{2}}$,

$(i=0,1,2,3)$. The difference between $\widetilde{B}_{0}$ and $B_{0}$ comes from the infrared singularity in $\widetilde{B}_{0}$. From $(1.30),(1.31)$
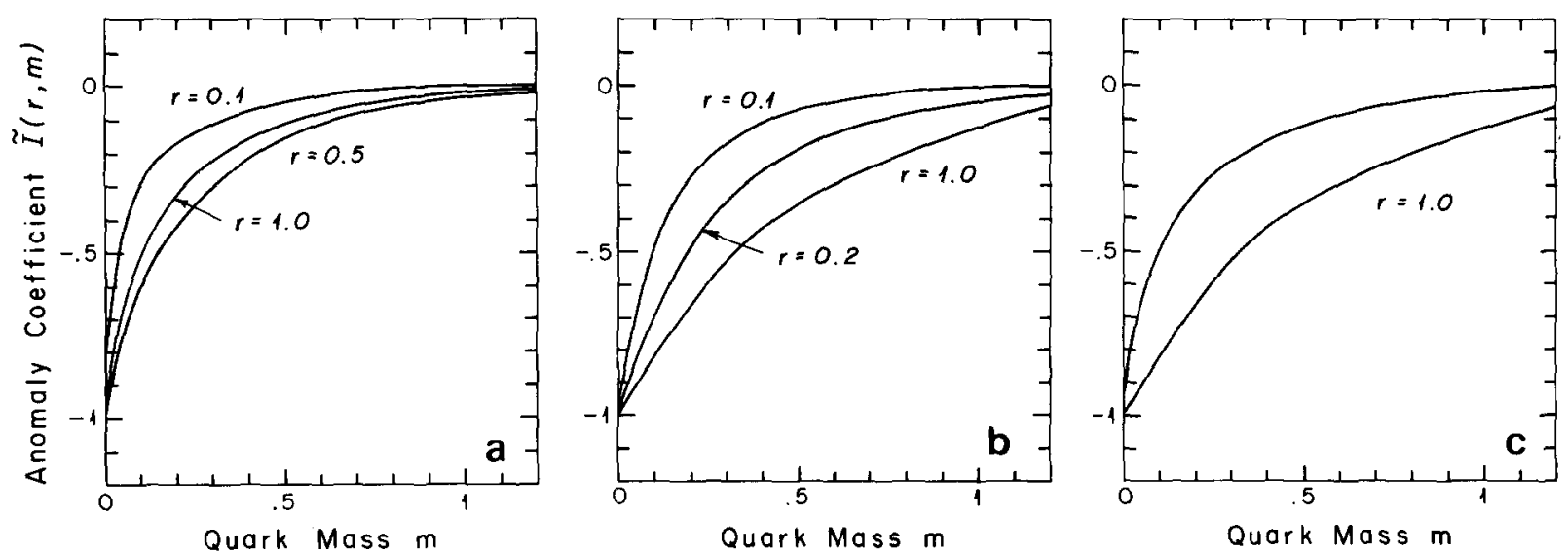

Fig. 2. (a) The behavior of the anomaly coefficient $\widetilde{I}(r, m)$ (see eq. (1.27)) for the Wilson action. (b) The behavior of $\widetilde{I}(r, m)$ for the improved action. (c) A comparison of $\widetilde{I}(r, m)$ for the Wilson and improved actions at $r=1$. 
Table 1

$\widetilde{I}(r, m)$ for the Wilson and improved action.

\begin{tabular}{lllll}
\hline & $m$ & $\tilde{I}(r, m)$ Wilson action & $\tilde{I}(r, m)_{\text {improved action }}$ \\
\hline 1.0 & 0.01 & -0.897 & -0.981 \\
& & 0.1 & -0.520 & -0.824 \\
& 0.2 & -0.329 & -0.675 \\
& 0.5 & -0.116 & -0.366 \\
& 1.0 & -0.0324 & -0.138 \\
& & 0.01 & -0.934 & -0.980 \\
& 0.1 & -0.628 & -0.818 \\
& 0.2 & -0.432 & -0.666 \\
& 0.5 & -0.162 & -0.358 \\
& 1.0 & -0.0402 & -0.129 \\
& & & -0.925 \\
& 0.1 & 0.01 & -0.897 & -0.496 \\
& 0.1 & -0.395 & -0.277 \\
& 0.2 & -0.190 & -0.0723 \\
& 0.5 & -0.0362 & -0.0139 \\
\hline
\end{tabular}

and (1.40) we obtain for $m \rightarrow 0$

$I(r)=0, \quad \tilde{I}(r)=-1$.

We have therefore shown that the improved action gives the correct anomaly in the continuum limit, and that the deviation in one loop level from the tree level axial Ward identity is zero.

In figs. $2 \mathrm{a}$ and $2 \mathrm{~b}$ we present the behavior of $\widetilde{I}(r, m)$ for the Wilson and improved fermion actions as a function of $r$ and $m$, respectively. A comparison between them shows (see fig. $2 c$ ) that the improved action gives a more reasonable value for the anomaly coefficient at finite $m$ than the Wilson action. Figs. $3 \mathrm{a}$ and $3 \mathrm{~b}$ also show the individual contributions of each fermion species to the anomaly. (See also table 1.)

Because of the connection in (large $N$ ) QCD between the anomaly and the $\eta^{\prime}$ mass, our calculation would suggest (if one assumes proportionality between $\widetilde{I}(m, r)$ and $\left.m_{\eta^{\prime}}\right)$ a strong suppression of the $\pi-\eta^{\prime}$ isospin splitting
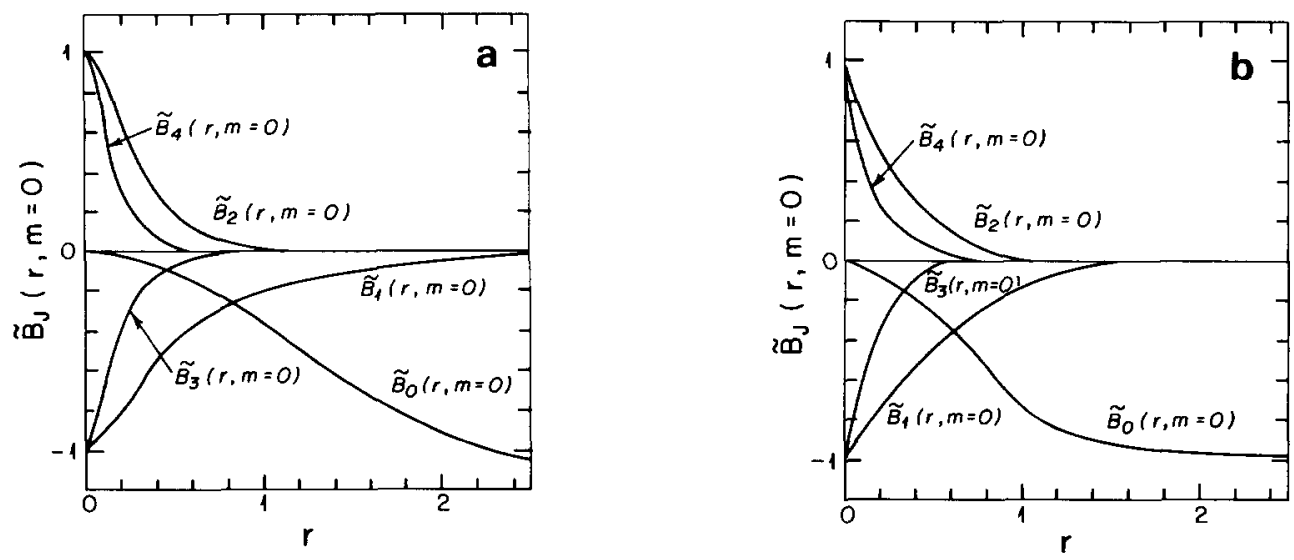

Fig. 3. (a) The behavior of $\widetilde{B}_{i}(r, m=0$ ) (see eq. (1.31)) for each fermion species for the Wilson action. (b) The behavior of $\widetilde{B}_{i}(r, m=0)$ for each fermion species for the improved action. 
for finite and not too small quark mass in the Wilson fermion case. When the improved action is used the splitting should increase significantly. A too small value for the $\eta^{\prime}$ mass was in fact suggested by recent numerical studies at $g^{2}=1[8]$.

2. Relationship between decay constants on the lattice and in the continuum. A set of quantities that can be easily computed by Monte Carlo simulation are the meson decay constants. It is known that the values of the meson decay constants on the lattice in the Wilson case are about a factor two larger than their experimental values [8]. The authors of ref. [9] have computed the first order perturbative correction that relates the continuum and lattice local operator in the Wilson case. But it appears that the corrections are still too small to compensate for the observed discrepancy.

Let us give here some predictions for these quantities in the case of the improved action. The relevant local operators used in Monte Carlo simulation to measure the meson masses and decay constants are of the form

$O_{i}(x)=\bar{\psi}_{1}(x) \Gamma_{i} \psi_{2}(x)$,

where $\psi_{1.2}(x)$ are the quark fields of flavor 1,2 and $\Gamma_{i}$ is one of the 16 Dirac matrices. The meson decay constants are defined as follows

$\left(m_{1}+m_{2}\right)\left\langle 0\left|\bar{\psi}_{2} \gamma_{5} \psi_{1}\right| \mathrm{P}\right\rangle=\sqrt{2} f_{\mathrm{P}} m_{\mathrm{P}}^{2}, \quad\left(m_{1}-m_{2}\right)\left\langle 0\left|\bar{\psi}_{2} \psi_{1}\right| \mathrm{S}\right\rangle=f_{\mathrm{S}} m_{\mathrm{S}}^{2}$,

$\left\langle 0\left|\bar{\psi}_{2} \gamma_{\mu} \psi_{1}\right| \mathrm{V}\right\rangle=f_{\mathrm{V}}^{-1} m_{\mathrm{V}}^{2} \epsilon_{\mu}, \quad\left\langle 0\left|\bar{\psi}_{2} \gamma_{5} \gamma_{\mu} \psi_{1}\right| \mathrm{A}\right\rangle=f_{\mathrm{A}}^{-1} m_{\mathrm{A}}^{2} \epsilon_{\mu}$,

where $m_{1,2}$ are the quark masses. P, S, V and A denote the pseudoscalar, scalar, vector and axial vector meson respectively.

The matrix elements of the currents on the LHS of eq. (2.2) depend on the regularization procedure. At one loop level one has:

$\left[\left(m_{1}+m_{2}\right)\left\langle 0\left|\bar{\psi}_{2} \gamma_{5} \psi_{1}\right| \mathrm{P}\right\rangle\right]_{\text {cont }}=\left(1+\left(\alpha_{\mathrm{s}} / 4 \pi\right) C_{\mathrm{F}} \Delta_{\mathrm{P}}\right)\left[\left(m_{1}+m_{2}\right)\left\langle 0\left|\bar{\psi}_{2} \gamma_{5} \psi_{1}\right| \mathrm{P}\right\rangle\right]_{\text {latt }}$,

$\left[\left\langle 0\left|\bar{\psi}_{2} \gamma_{\mu} \psi_{1}\right| \mathrm{V}\right\rangle\right]_{\mathrm{cont}}=\left(1+\left(\alpha_{\mathrm{s}} / 4 \pi\right) C_{\mathrm{F}} \Delta_{\mathrm{V}}\right)\left[\left\langle 0\left|\bar{\psi}_{2} \gamma_{\mu} \psi_{1}\right| \mathrm{V}\right\rangle\right]_{\text {latt }}$

and similarly for the scalar and axial vector mesons $\left(\Delta_{\mathrm{S}}\right.$ and $\left.\Delta_{\mathrm{A}}\right)$. The coefficients $\Delta_{\mathrm{S}, \mathrm{P}, \mathrm{V}, \mathrm{A}}$ can be computed in perturbation theory. By calculating the $O\left(\alpha_{s}\right)$ diagrams for the vertex correction and quark self energy (see fig. 4) on the lattice and in the continuum we get

$\Delta_{\mathrm{S}, \mathrm{P}}=\Delta_{1, \gamma_{5}}+\Delta_{\Sigma_{2}}, \quad \Delta_{\mathrm{V}, \mathrm{A}}=\Delta_{\gamma_{\mu}, \gamma_{5} \gamma_{\mu}}+\Delta_{\Sigma_{1}}$,

where

$$
\begin{aligned}
& \Delta_{1, \gamma_{5}}=4\left(\gamma-F_{0001}-1\right)+4 \pi^{2}\left(I_{1} \pm I_{2}-I_{3}\right), \quad \Delta_{\gamma_{\mu}, \gamma_{5} \gamma_{\mu}}=\gamma-F_{0001}-2+4 \pi^{2}\left(\frac{1}{4} I_{1} \mp \frac{1}{2} I_{2}-I_{3}\right), \\
& \Delta_{\sigma_{\mu, \nu}}=-4 \pi^{2} I_{3},
\end{aligned}
$$

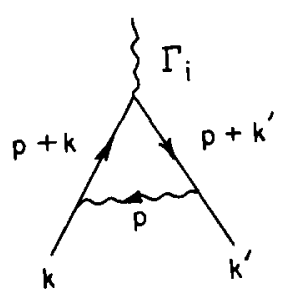

a

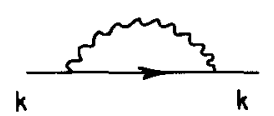

b

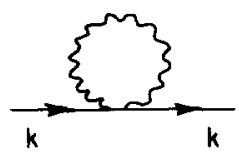

Fig. 4. (a), (b) One-loop diagrams for the vertex correction and the quark self energy. 
Table 2

Some values of $\Delta$.

\begin{tabular}{rccccccrr}
$r$ & $\Delta_{\Sigma_{1}}$ & $\Delta_{\Sigma_{2}}$ & $\Delta_{1}$ & $\Delta_{\gamma_{5}}$ & $\Delta_{\gamma_{\mu}}$ & $\Delta_{\gamma_{5} \gamma_{\mu}}$ & $\Delta_{\sigma_{\mu \nu}}$ \\
\hline 0.0 & -3.95 & 10.6 & -26.6 & -26.6 & -7.66 & -7.66 & 0 \\
0.2 & -4.17 & 9.18 & -17.5 & -22.6 & -7.63 & -5.09 & -0.44 & -0.98 \\
0.4 & -4.75 & 6.16 & -10.6 & -18.2 & -7.23 & -3.45 & -1.34 & -1.59 \\
0.6 & -5.53 & 3.13 & -7.44 & -15.1 & -6.75 & -2.91 & -2.76 & -1.75 \\
0.8 & -6.42 & 0.46 & -5.85 & -12.9 & -6.32 & -2.74 & -1.9 \\
\hline
\end{tabular}

with

$I_{1}=\int_{-\pi}^{\pi} \frac{\mathrm{d}^{4} p}{(4 \pi)^{4}}\left[\frac{\Delta_{8}}{4 \Delta_{1}^{3}}-\frac{\Delta_{5} \Delta_{7}}{\Delta_{2}^{2} \Delta_{1}}\right], \quad I_{2}=4 r^{2} \int_{-\pi}^{\pi} \frac{\mathrm{d}^{4} p}{(2 \pi)^{4}} \frac{1}{\Delta_{2}^{2} \Delta_{1}}\left[\frac{16}{9} \Delta_{3}^{2} \Delta_{5}-\frac{4}{3} \Delta_{3} \Delta_{4}+\frac{1}{4} \Delta_{6} \Delta_{7}\right]$,

$I_{3}=\int_{-\pi}^{\pi} \frac{\mathrm{d}^{4} p}{(2 \pi)^{4}} \frac{1}{\Delta_{2}^{2} \Delta_{1}}\left[\frac{16}{3} r^{2} \Delta_{3} \Delta_{4}+\frac{64}{9} r^{4} \Delta_{3}^{2} \Delta_{6}\right]$

$\Delta_{1}=\sum_{\rho} \sin ^{2} \frac{1}{2} p_{\rho}, \quad \Delta_{2}=\left(\frac{8}{3} r \sum_{\rho} \sin ^{4} \frac{1}{2} p_{\rho}\right)^{2}+\frac{16}{9} \sum_{\rho}\left(\sin p_{\rho}-\frac{1}{8} \sin 2 p_{\rho}\right)^{2}, \quad \Delta_{3}=\sum_{\rho} \sin ^{4} \frac{1}{2} p_{\rho}$,

$\Delta_{4}=\left(\frac{4}{3}\right)^{3} \sum_{\rho}\left(\cos \frac{1}{2} p-\frac{1}{4} \cos p\right)_{\rho}\left(\sin \frac{1}{2} p-\frac{1}{2} \sin p\right)_{\rho}\left(\sin p-\frac{1}{8} \sin 2 p\right)_{\rho}, \quad \Delta_{5}=\left(\frac{4}{3}\right)^{2} \sum_{\rho}\left(\cos \frac{1}{2} p-\frac{1}{4} \cos p\right)_{\rho}^{2}$,

$\Delta_{6}=\left(\frac{4}{3}\right)^{2} \sum_{\rho}\left(\sin \frac{1}{2} p-\frac{1}{2} \sin p\right)_{\rho}^{2}, \quad \Delta_{7}=\left(\frac{4}{3}\right)^{2} \sum_{\rho}\left(\sin p-\frac{1}{8} \sin 2 p\right)_{\rho}^{2}, \quad \Delta_{8}=\sum_{\rho} \sin ^{2} p_{\rho}$,

and $\gamma$ is Euler's constant. The quantity $F_{0001}=1.3109$ is defined in ref. [10]. From eqs. (2.4)-(2.6) and the expression for $\Sigma_{1}$ and $\Sigma_{2}$ given in ref. [1]

$\Delta_{\Sigma_{1,2}}=\Sigma_{1,2}^{\text {cont }}-\Sigma_{1,2}^{\text {lat } t}$

we obtain numerically the correction coefficients. These are shown in table 2 . From the numbers in table 2 we finally get

$f_{\pi}=\left[1+\left(\alpha_{\mathrm{s}} / 4 \pi\right) C_{\mathrm{F}} \Delta_{\pi}\right] f_{\pi}^{\mathrm{latt}}=\left(1-0.112 g^{2}\right) f_{\pi}^{\mathrm{latt}}$,

$f_{\rho}^{-1}=\left[1+\left(\alpha_{\mathrm{s}} / 4 \pi\right) C_{\mathrm{F}} \Delta_{\rho}\right]\left(f_{\rho}^{-1}\right)^{\mathrm{latt}}=\left(1-0.112 g^{2}\right)\left(f_{\rho}^{-1}\right)^{1 \mathrm{att}}$

for $r=1$ and SU(3).

3. Conclusions. In this paper we have presented some analytic results for the axial vector current anomaly and the finite renormalizations of local currents on the lattice for the improved fermion action. This allowed us to investigate the regularization dependence of the anomaly and discuss the possible relevance of our results for the problem of computing the $\eta^{\prime}$ mass in lattice QCD. Since we have found the anomaly coefficient for finite quark mass to depend significantly on the fermion action regularization, we expect a similar situation to arise also in the case of the $\eta^{\prime}$ mass.

This research was supported by the U.S. Department of Energy under grant no. DE-AC02-76ER02220. 


\section{References}

[1] H. Hamber and C.M. Wu, Phys. Lett. 133B (1983) 351.

[2] K.G. Wilson, Phys. Rev. D10 (1974) 2445; in: New phenomena in subnuclear physics (Erice, 1975), ed. A. Zichichi (Plenum, New York, 1977).

[3] S.L. Adler, Phys. Rev. 177 (1969) 2426;

J.S. Bell and R. Jackiw, Nuovo Cimento 60A (1969) 47;

S.L. Adler, Brandeis University Summer Institute in Theoretical Physics, eds. S. Deser, M. Grisaru and H. Pendleton (MIT press, Cambridge, MA, 1970);

R. Jackiw, in: Lectures on current algebra and its applications (Princeton U.P., Princeton, 1972).

[4] L.H. Karsten and J. Smith, Nucl. Phys. B183 (1981) 103.

[5] W. Kerler, Phys. Rev. D23 (1981) 2383;

H. Kawai, R. Nakayama and K. Seo, Nucl. Phys. B189(1981) 40;

E. Seiler and I.O. Stamatescu, Phys. Rev. D25 (1982) 2177.

[6] N. Kawamoto and K. Shigemoto, Phys. Lett. 120B (1983) 183; preprint NBI-HE-82-47 (1982).

[7] S. Drell, M. Weinstein and S. Yankielowicz, Phys. Rev. D14 (1976) 1627;

B. Svetitsky, S. Drell, H. Quinn and M. Weinstein, Phys. Rev. D22 (1980) 490, 1190.

[8] H. Hamber, in: Proc. VIth Johns Hopkins Workshop on Current Problems in Particle Theory (Florence, 1982) (Johns Hopkins University Press, 1982);

H. Hamber and G. Parisi, Phys. Rev. D27 (1983) 208.

[9] B. Meyer and C. Smith, Phys. Lett. 123B (1982) 62;

G. Martinelli and Y.C. Zhang, Phys. Lett. 123B (1983) 433.

[10] A. Gonzales-Arroyo and C.P. Korthals Altes, Nucl. Phys. B20S [FS5] (1983) 46. 